\title{
molecules
}

ISSN 1420-3049

(C) 2008 by MDPI

www.mdpi.org/molecules

Full Paper

\section{Synthesis and Characterization of Konjac Glucomannan-Graft-Polyacrylamide via $\gamma$-Irradiation}

\author{
Zhenlin Xu ${ }^{1}$, Youhui Yang ${ }^{1}$, Yueming Jiang ${ }^{2}$, Yuanming Sun ${ }^{1, *}$, Yudong Shen ${ }^{1}$ \\ and Jie Pang ${ }^{1,3}$ \\ ${ }^{1}$ College of Food Science, South China Agricultural University, Guangzhou 510642, P. R. China; \\ E-mails: jallent@163.com; yhyang@scau.edu.cn; shenyudong@scau.edu.cn \\ ${ }^{2}$ South China Botanical Garden, Chinese Academy of Sciences, Guangzhou 510650, P. R. China; \\ E-mail: ymjiang@scbg.ac.cn \\ ${ }^{3}$ College of Food Science, Fujian Agriculture and Forestry University, Fuzhou 350002, R. China; \\ E-mail: pang3721941@163.com
}

* Author to whom correspondence should be addressed; E-mail: ymsun@scau.edu.cn

Received: 16 January 2008; in revised form: 20 February 2008 / Accepted: 20 February 2008 / Published: 1 March 2008

\begin{abstract}
The synthesis of konjac glucomannan-graft-polyacrylamide (KGM-g-PAM) was carried out at $25^{\circ} \mathrm{C}$ by $\gamma$-irradiation under a $\mathrm{N}_{2}$ atmosphere. The effects of absorbed radiation dosage and monomer concentration on grafting yield and water absorbency were studied. The grafted copolymers were characterized using Fourier Transform Infrared (FTIR) spectroscopy, nuclear magnetic resonance (NMR), x-ray diffraction (XRD), thermogravimetric analysis (TGA) and gel permeation chromatography (GPC). The grafting yield was observed to increase with increasing absorbed dosage and monomer concentration. Compared with the original KGM, the grafted copolymers exhibited better thermal stability and water absorbency. The results suggest that $\gamma$-irradiation is convenient and efficient for inducing graft copolymerization of KGM and acrylamide (AM).
\end{abstract}

Keywords: Konjac glucomannan, polyacrylamide, graft copolymerization, $\gamma$-irradiation 


\section{Introduction}

Konjac glucomannan (KGM) is a type of neutral heteropolysaccharide extracted from tubers of Amorphophallus konjac C. Koch. Chemically, KGM has $\beta$ - $(1 \rightarrow 4)$ linked $D$-mannose and $D$-glucose units in a molar ratio of 1.6:1as the main chain, with branches joined through C-3 of the $D$-glucosyl and $D$-mannosyl residues and a low number of acetyl groups (approximately one acetyl group per 17 residues) at the C-6 position [1-3]. The chemical structure of KGM is shown in Figure 1. KGM has long been used as a health food in China and Japan. Due to its characteristics of low cost, high viscosity, excellent film-forming ability, good biocompatibility and biodegradability, as well as gelforming properties, KGM and its derivatives have been used widely in various fields, such as food and food additives, and the pharmaceutical, biotechnology and fine chemical industries [4].

Figure 1. The chemical structure of konjac glucomannan.

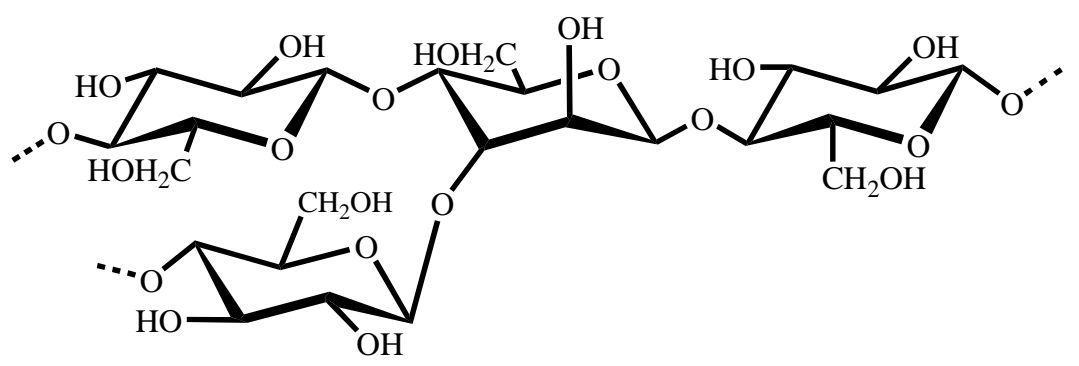

Graft copolymerization of natural polysaccharides such as chitosan, carrageenan and guar is becoming an important resource for developing advanced materials as it can improve the functional properties of natural polysaccharides [5-7]. The synthesis of grafted copolymers is usually conducted by the conventional redox grafting method or high-energy radiation including microwave, UV ray, $\gamma$ ray and electron beam [8-10]. Of these methods, ${ }^{60} \mathrm{Co} \gamma$-irradiation exhibits the most potential to synthesize the grafted copolymers, due to the higher energy emission, simpler preparation and lower cost [11]. $\gamma$-Irradiation is an ionic, non-thermal processing technology which continues to receive attention as a preservation and functional modification agent in polymer research and application [12]. With regard to its safety, a Joint FAO/IAEA/WHO Expert Committee on Food Irradiation (JECFI) declared in 1997 that food irradiated to any dosage appropriate to achieve the intended technological objective was both safe to consume and nutritionally adequate [13].

The synthesis of KGM with vinyl monomer for colon-specific drug delivery and as a superabsorbent by the conventional redox grafting method has been reported [14,15]. In our previous work we examined the effect of $\gamma$-irradiation on the properties of KGM and suggested that it was convenient to modify KGM [16]. However, little information on graft copolymerization of KGM and acrylamide (AM) by $\gamma$-irradiation is available. The objective of the present work was to investigate the feasibility of graft copolymerization of KGM and AM by $\gamma$-irradiation. The effects of absorbed dosage and monomer concentration on grafting yield and water absorbency and the properties of the grafted products were investigated. 


\section{Results and Discussion}

Synthesis and properties

The synthesis of KGM-g-PAM was conducted by a simultaneous irradiation method, as described by Biswal et al. [7]. The effects of absorbed dosage and monomer concentration on grafting yield and water absorbency of the grafted copolymers were investigated. Figure 2 shows the effect of total absorbed dosage on the grafting yield and water absorbency of KGM-g-PAM. The grafting yield increased with irradiation dosage. A high grafting yield ( $>100 \%)$ could be achieved at a dosage above $0.8 \mathrm{kGy}$ and it was $213.1 \%$ at a dosage of $3.2 \mathrm{kGy}$. However, the water absorbency of the grafted copolymer increased with increasing dosage at first and then decreased slightly when the dosage was higher than $1.6 \mathrm{kGy}$. The water absorbency was $217 \mathrm{~g} / \mathrm{g}$ at the dosage of $1.6 \mathrm{kGy}$. The increasing grafting yield with increasing dosage was mainly attributed to the increasing presence of radicals induced by higher irradiation dosage, as first described by Chapiro [17]. However, the results indicated that water absorbency of the grafted copolymer was not always positively associated with grafting yield. This might be explained by the Flory's theory [18]: there is an appropriate crosslink density for the maximal water absorbency. The increased grafting yield might improve the crosslink density of the copolymers, which resulting in a difficulty to absorb water. Luo et al. [19] and Huacai et al. [9] also reported that higher irradiation dosage could decrease the water absorbency of graft copolymers.

Figure 2. Effect of absorbed dosage on grafting yield and water absorbency of KGM-g-PAM (grafting condition: $[\mathrm{KGM}]=0.6 \%$, $[\mathrm{AM}]=0.3 \mathrm{~mol} / \mathrm{L})$.

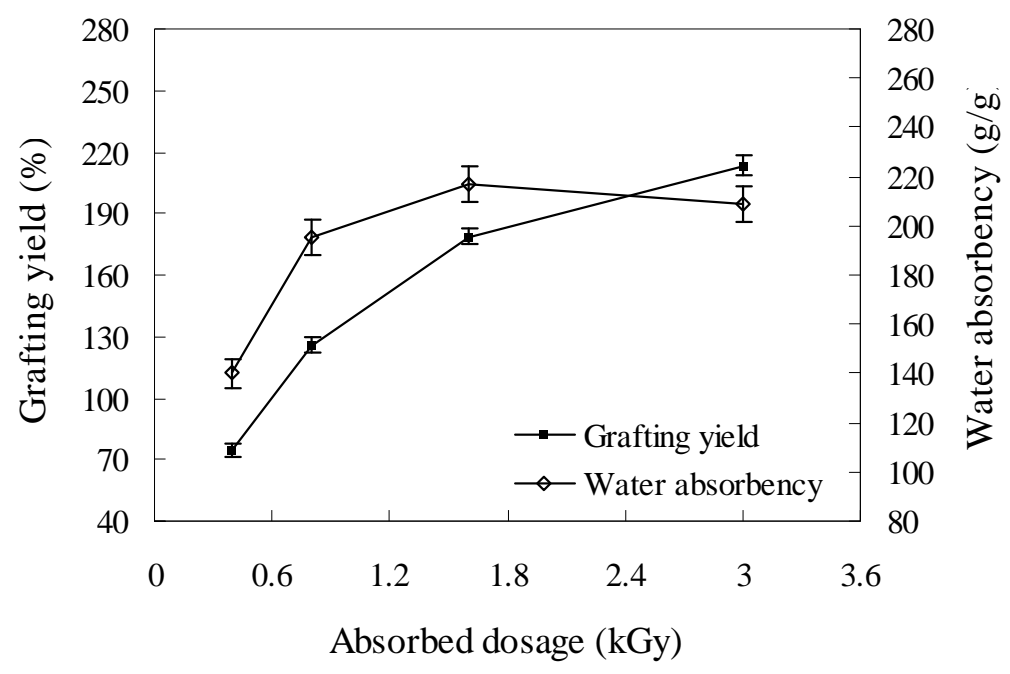

Figure 3 illustrates the effects of (a) KGM concentration and (b), AM concentration on grafting yield and water absorbency of the grafted copolymer. Increases in both KGM and AM contents could enhance grafting yield, but the concentration of AM played a more important role in the grafting yield than that of KGM. This may due to the inherent properties of KGM and AM upon irradiation exposure: whereas AM predominantly undergoes crosslinking [20], KGM, like other natural polymers such as carrageenan and alginates [21,22], undergoes degradation [16, 23]. Moreover, the G value for radical 
formation of AM on $\gamma$-irradiation was reported to be much higher than that of natural polymers [24,25]. Therefore, the higher $\mathrm{G}$ value and predominant crosslinking nature of AM on irradiation both favored faster grafting with increase in monomer concentration. The water absorbency of grafted copolymer increased with increase of grafting yield at first and then decreased slightly.

Figure 3. Effects of (a) KGM concentration; grating condition: dosage $=0.8 \mathrm{kGy},[\mathrm{AM}]=0.3 \mathrm{~mol} / \mathrm{L})$ and $(\mathrm{b}), \mathrm{AM}$ concentration; grafting condition: dosage $=0.8 \mathrm{kGy},[\mathrm{KGM}]=0.6 \%$ ) on grafting yield and water absorbency of KGM-g-PAM.
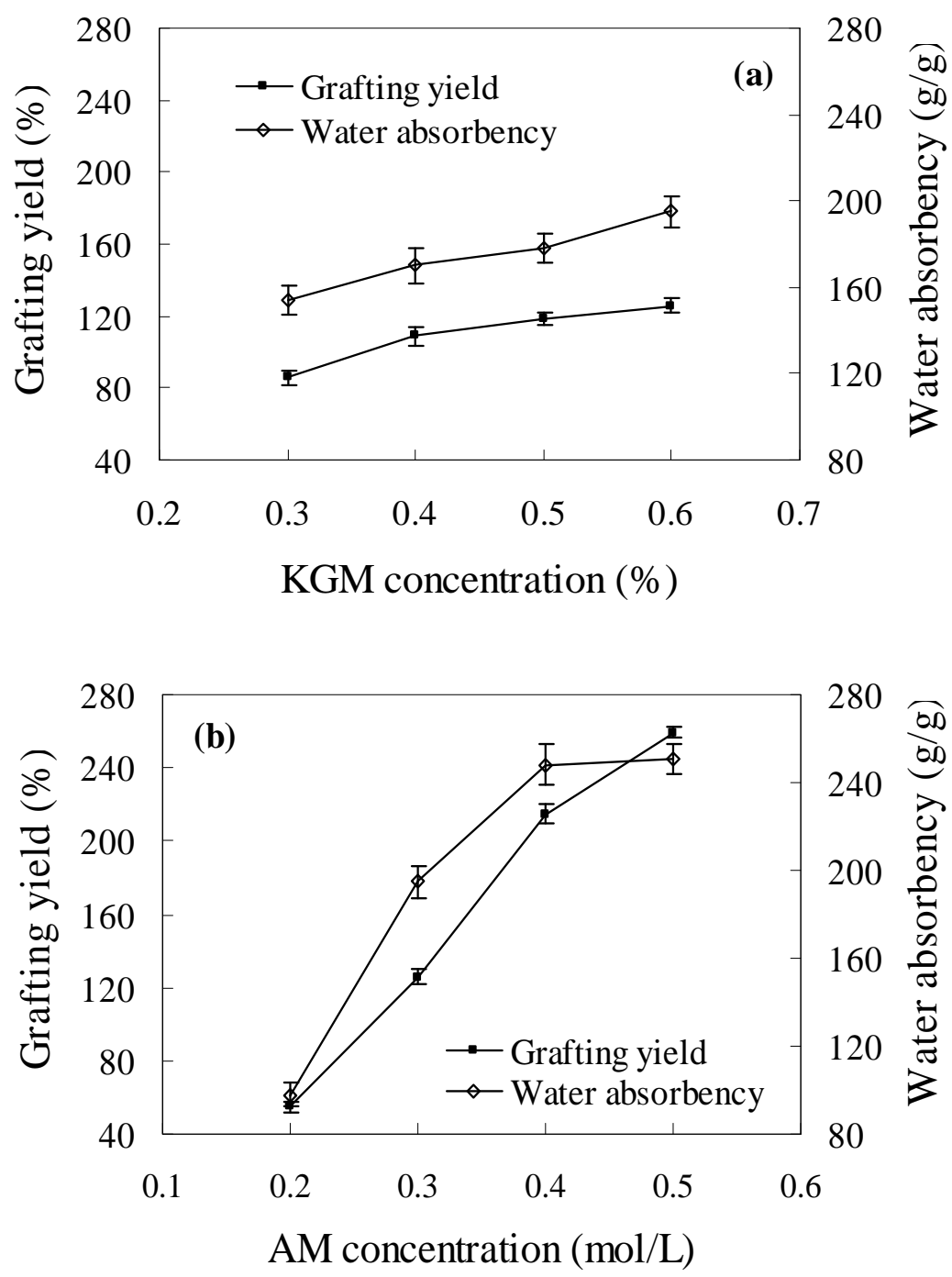

\section{Characterization}

The characteristic FTIR absorption bands of the original KGM and grafted copolymer are presented in Figure 4. The main characteristic peaks of KGM were at $3400 \mathrm{~cm}^{-1}$ (O-H stretch), 2887 $\mathrm{cm}^{-1}$ (C-H stretch), $1736 \mathrm{~cm}^{-1}$ (C=O stretch), $1164 \mathrm{~cm}^{-1}$ (bridge $\mathrm{O}$ stretch), and $1092 \mathrm{~cm}^{-1}(\mathrm{C}-\mathrm{O}$ stretch). 
Figure 4. FTIR spectra of KGM and KGM-g-PAM.

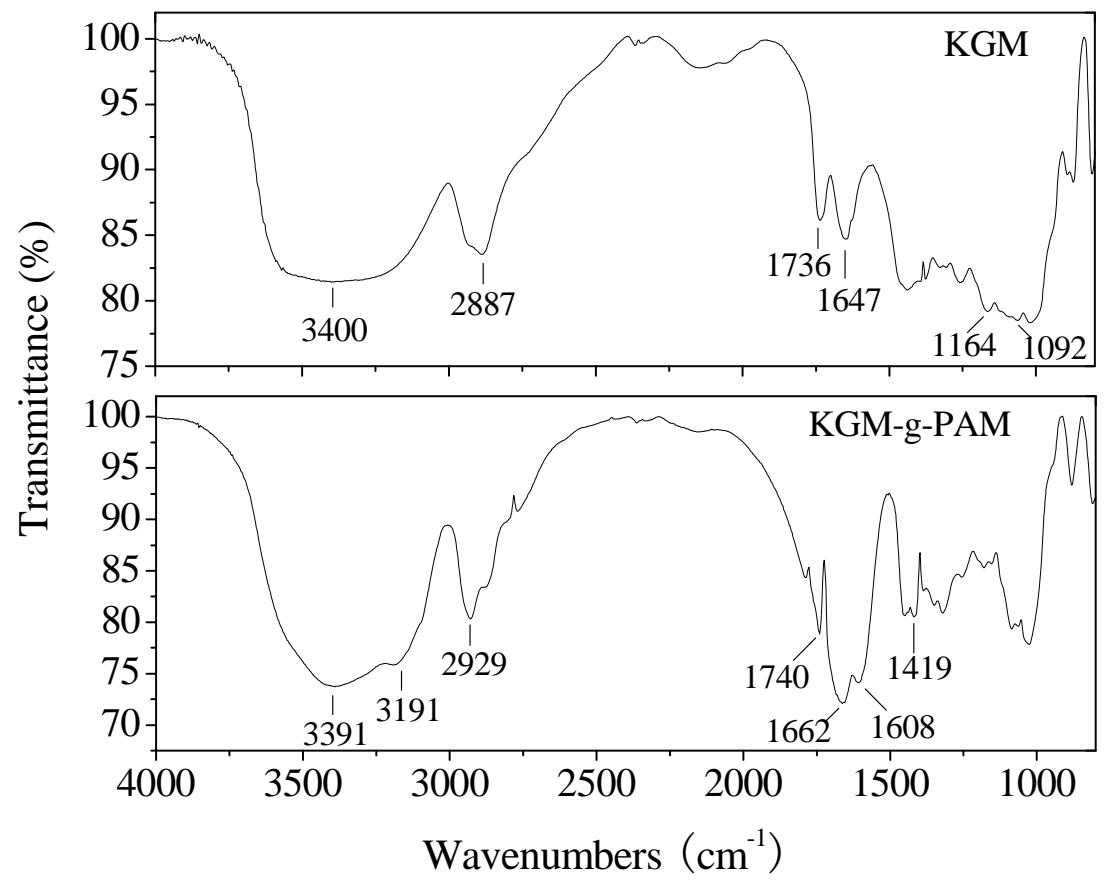

The result was similar to the reports of Huacai et al. [9] and Yu et al. [26]. In the spectra of the grafted copolymer, in addition to the KGM characteristic peaks, peaks at 3139 and $1662 \mathrm{~cm}^{-1}$ indicated the $\mathrm{N}-\mathrm{H}$ stretching and $\mathrm{N}-\mathrm{H}$ bending of the amide bands, which are characteristic of the $-\mathrm{CONH}_{2}$ group present in the acrylamide. In addition, a peak at $1419 \mathrm{~cm}^{-1}$ was assigned to $\mathrm{C}-\mathrm{N}$ stretching. These changes provided strong evidence supporting the grafting of acrylamide onto the KGM.

Figure 5 shows the ${ }^{13} \mathrm{C}$-NMR spectra of KGM and its grafted copolymers. According to the studies on the NMR spectra of KGM by Vieira and Gil [27] and Katsuraya et al. [28], the overlapping peaks in the 71-80 ppm region were assigned to the pyranose ring, including the presence of glucosyl (G) and mannosyl (M) units. The peak at about 63 ppm can be assigned as $\mathrm{C} 6$ of both $\mathrm{G}$ and $\mathrm{M}$ units, while the $\mathrm{C} 1$ of both $\mathrm{G}$ and $\mathrm{M}$ units was observed at 102-106 ppm. For KGM-g-PAM, the presence of a very intense peak at $182.1 \mathrm{ppm}$ was due to the polyacrylamide carbonyl groups. The two peaks at about $37 \mathrm{ppm}$ and $44 \mathrm{ppm}$ were assigned as the methylene group and the carbon connected to carbonyl group of polyacrylamide, respectively [29]. The NMR spectra results thus further confirmed the FTIR results.

X-ray diffractograms of KGM and KGM-g-PAM are shown in Figure 6. The XRD pattern of KGM showed a weak but broad reflection falling at about $2 \theta=20^{\circ}$. For the grafted copolymer, the peak at $2 \theta=20^{\circ}$ decreased significantly, indicating that the conjugation of KGM with PAM reduced the crystallinity of KGM. KGM was reported to have a hydrated crystal of low crystallinity [30] and PAM to be an amorphous material, which did not show any significant crystallinity peak [31]. The decrease of crystallinity might due to the intra- and intermolecular- hydrogen bonding interactions between KGM and PAM, which destroyed the original molecular orientation of KGM. Xiao et al. [32] also found that the graft copolymerization of KGM and PAM could lower the crystallinity of KGM, which suggested that KGM and PAM chains were mixed well at a molecular level. 
Figure 5. ${ }^{13} \mathrm{C}-\mathrm{NMR}$ spectra of KGM and KGM-g-PAM.

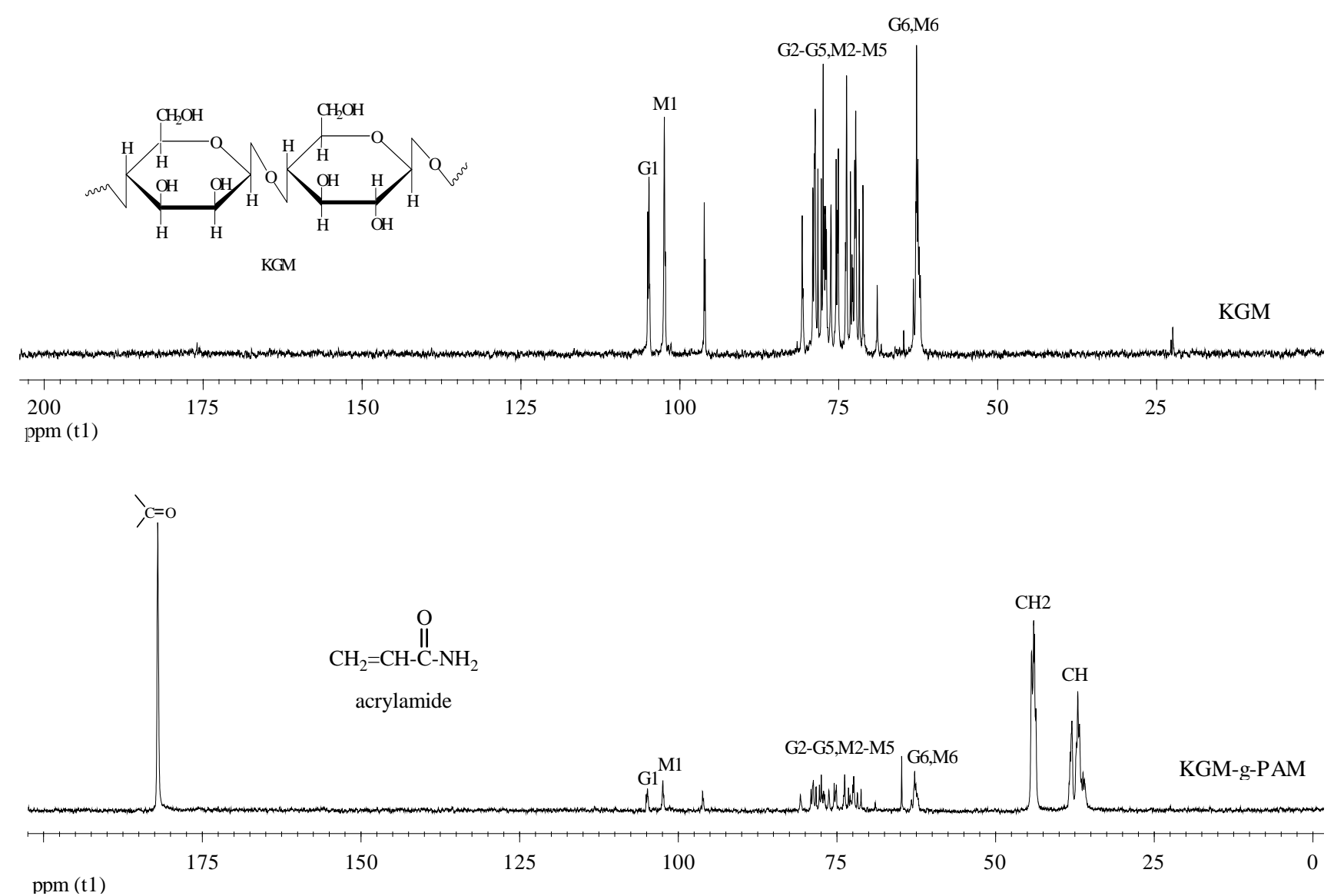

Figure 6. XRD patterns of KGM and KGM-g-PAM.

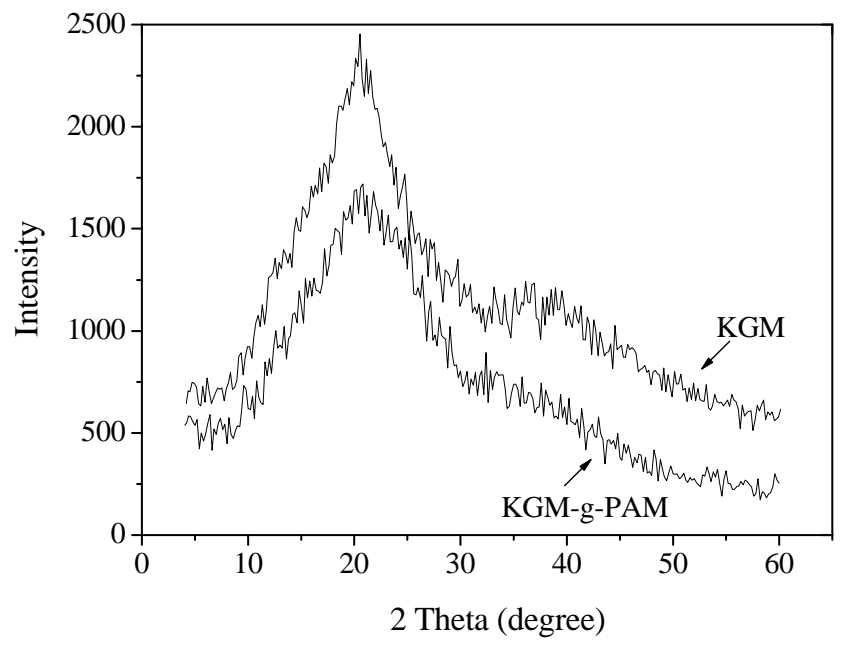

The thermogravimetric analysis (TGA) technique was employed to characterize the thermal properties of KGM and KGM-g-PAM; the results are shown in Figure 7. The decomposition of KGM started at about $250^{\circ} \mathrm{C}$. The weight loss rate increased with increasing temperature up to $296^{\circ} \mathrm{C}$ and then decreased. Nearly $61 \%$ of KGM degraded at the first step and about $0.5 \%$ survived up to $550^{\circ} \mathrm{C}$. However, the TGA profile of KGM-g-PAM was distinctly different from that of KGM. 
Figure 7. TG and DTG curves of KGM and KGM-g-PAM.

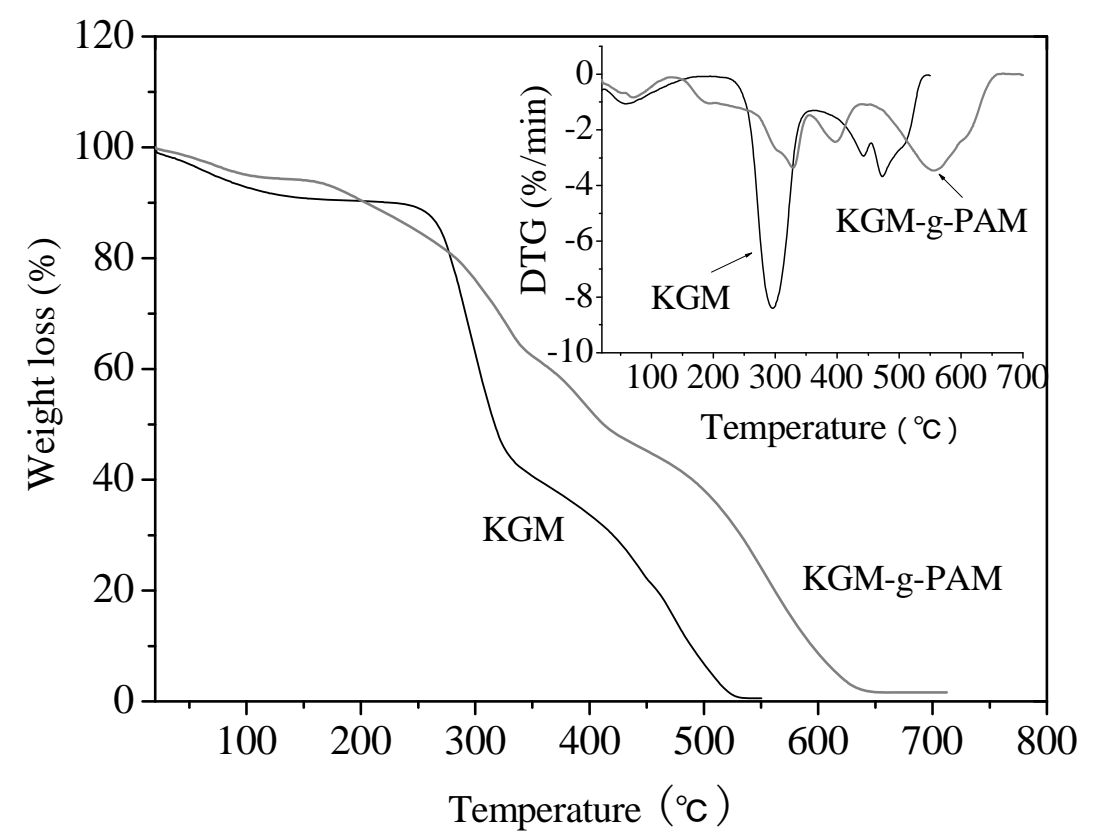

The grafted copolymers had a continuous weight loss with increasing temperature and began to degrade at about $180^{\circ} \mathrm{C}$, with the maximum weight loss rate occurring at $328^{\circ} \mathrm{C}$. Furthermore, the weight loss rate of the KGM-g-PAM was slower than that of $\mathrm{KGM}$ at above $276^{\circ} \mathrm{C}$ and about $1.6 \%$ still existed at $700^{\circ} \mathrm{C}$. The results indicated that the initial decomposition temperature $\left(T_{\mathrm{i}}\right)$ of KGM-gPAM was lower than that of KGM, and the maximum decomposition temperature $\left(T_{\mathrm{m}}\right)$ and final decomposition temperature $\left(T_{\mathrm{f}}\right)$ were higher than that of KGM. The previous reports also revealed that the graft copolymerization of natural polysaccharides with vinyl monomer could enhance their thermal stability [7,9,29].

The molecular weights of KGM and its grafted copolymer were determined by GPC. The elution curves are presented in Figure 8. The peak molecular mass $\left(M_{\mathrm{p}}\right)$ of the grafted copolymer decreased significantly and the weight average molecular mass $\left(M_{\mathrm{w}}\right)$ and number average molecular mass $\left(M_{\mathrm{n}}\right)$ decreased slightly compared with that of KGM (Table 1). The elution curve of KGM-g-PAM was smoother than that of KGM, which could be a reflection of the decrease in polydispersity. The slight decrease of molecular weight might be attributed to the degradation of KGM expose to irradiation. The results were in agreement with some earlier reports [33,34].

\section{Conclusions}

The synthesis and characterization of konjac glucomannan-graft-polyacrylamide via $\gamma$-irradiation was studied. The results indicated that $\gamma$-irradiation was convenient for inducing graft copolymerization of KGM and AM at low dosage and obtaining graft copolymers with high grafting yield (>100\%). The grafting yield increased with increasing dosage and monomer concentration, while the water absorbency increased first and then decreased slightly with increasing dosage and monomer concentration. FTIR, NMR and XRD analyses showed that AM had been grafted onto KGM successfully. TGA results indicated that the grafted copolymer exhibited better thermal stability in 
comparison with original KGM. The molecular weight of grafted copolymer decreased slightly according to the results of GPC.

Figure 8. GPC elution curves of KGM and KGM-g-PAM.
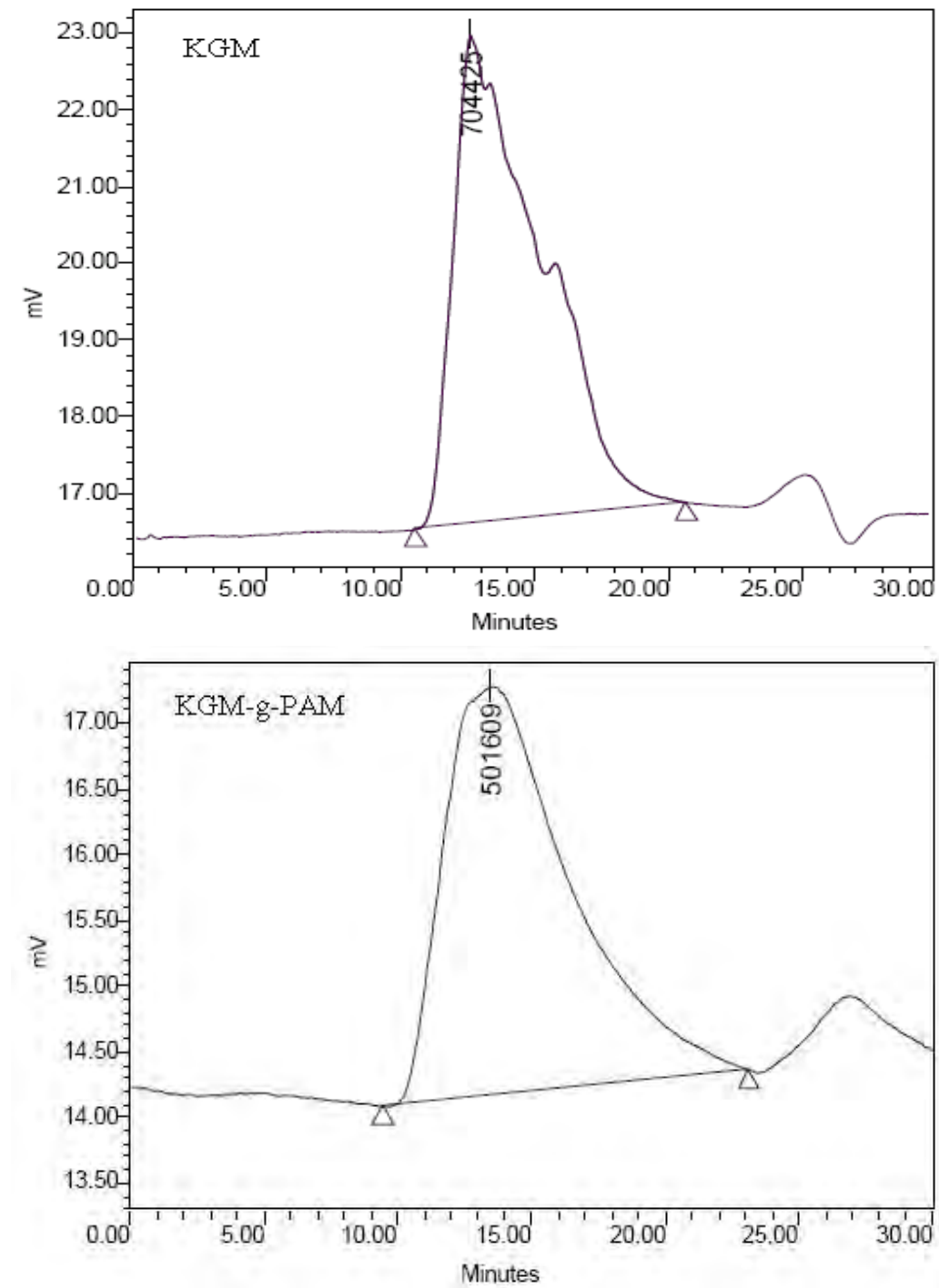

Table 1. The molecular weight and polydispersity of KGM and KGM-g-PAM

\begin{tabular}{lccc}
\hline Sample & $\boldsymbol{M}_{\mathbf{w}}$ & $\boldsymbol{M}_{\mathbf{n}}$ & Polydispersity $\left(\boldsymbol{M}_{\mathbf{w}} / \boldsymbol{M}_{\mathbf{n}}\right)$ \\
\hline KGM & $4.81 \times 10^{5}$ & $3.33 \times 10^{5}$ & 1.441 \\
KGM-g-PAM & $4.28 \times 10^{5}$ & $3.02 \times 10^{5}$ & 1.418 \\
\hline
\end{tabular}

\section{Experimental}

\section{Materials}

KGM was obtained from Sanai Konjac Food Corp., Yibin, P.R. China. It was purified by washing several times with aqueous methanol. All the other regents were of analytical grade and used without further purification. Double-distilled water was used for these experiments. 


\section{Graft copolymerization}

KGM (0.6 g) was dissolved in water $(90 \mathrm{~mL})$ in a glass tube and kept stirring for $12 \mathrm{~h}$. AM solution ( $3 \mathrm{~mol} / \mathrm{L}, 10 \mathrm{~mL}$ ) was then added to the solution with stirring and stirring was continued for another $2 \mathrm{~h}$. The solutions were deoxygenated by nitrogen bubbling and then irradiated for 20, 40, 80 and $150 \mathrm{~min}$ at a fixed dosage rate of $20 \mathrm{~Gy} / \mathrm{min}$ at $25^{\circ} \mathrm{C}$. The irradiated solution was placed overnight for a complete reaction and then precipitated in excess of ethanol. The precipitate was dried and washed several times with a 3:2 mixture of acetic acid-dimethylformamide to remove the unreacted monomer and homopolymer. Finally, it was washed with ethanol and then dried at $55^{\circ} \mathrm{C}$ under vacuum.

\section{Determination of grafting yield and water absorbency}

The grafting yield was determined gravimetrically using the following equation:

$$
\text { Grafting yield }(\%)=\left[\left(W_{\mathrm{g}}-W_{\mathrm{o}}\right) / W_{\mathrm{o}}\right] \times 100
$$

where $W_{\mathrm{g}}$ and $W_{\mathrm{o}}$ were the weight of KGM after grafting and the initial weight of KGM, respectively.

The dried sample $(1.0 \mathrm{~g})$ was dispersed in water $(800 \mathrm{~mL})$ and kept stirring for at least $12 \mathrm{~h}$ at $25^{\circ} \mathrm{C}$ to reach swelling equilibrium, then the residual water was removed by filtration through a 100-mesh stainless steel screen. Water absorbency was calculated using the following equation:

$$
\text { Absorbency }(\mathrm{g} / \mathrm{g})=\left(W_{1}-W_{0}\right) / W_{0}
$$

where $W_{1}$ and $W_{0}$ are the weight of the water-swollen and the dry sample, respectively.

\section{Characterization}

Fourier transformed infrared spectroscopy (FTIR) measurements were performed on a Vector33 FTIR spectrometer (Bruker, Germany). Samples were mixed with $\mathrm{KBr}$ and then scanned against a blank KBr pellet background. The ${ }^{13} \mathrm{C}$-NMR spectra were recorded on a Bruker Model Avance DRX400 spectrometer at about $30^{\circ} \mathrm{C}$. X-ray diffraction (XRD) patterns were obtained with a D/max-IIIA xray diffractometer at the scattering angle $(2 \theta)$ of $4^{\circ}$ to $60^{\circ}$ and the scanning rate of $10^{\circ} / \mathrm{min}$. Thermogravimetric analysis (TGA) was conducted with a Netzsch TG 209 analyzer, the scan was carried out at a heating rate of $10.0^{\circ} \mathrm{C} / \mathrm{min}$ from 20 to $700{ }^{\circ} \mathrm{C}$. The molecular weights and molecular weight distributions were determined by 515-gel permeation chromatography (GPC). Unless otherwise stated, the grafting yield of samples used for analysis, which were synthesized under the following condition: absorbed dosage $=0.8 \mathrm{kGy},[\mathrm{KGM}]=0.6 \%,[\mathrm{AM}]=0.3 \mathrm{~mol} / \mathrm{L}$, was $125.94 \%$.

\section{Acknowledgements}

The authors are grateful for the financial support of the National Natural Science Foundation of China (Grant Nos. 30070533, 30471218 and 30371009). 


\section{References}

1. Smith, F.; Srivastava, H.C. Constitution studies on the glucomannan of konjac flower. J. Am. Chem. Soc. 1959, 81, 1715-1718.

2. Kato, K.; Matsuda, K. Studies on the chemical structure of konjac mannan. Agr. Biol. Chem. 1969, 33, 1446-1453.

3. Maeda, M.; Shimahara, H; Sugiyama, N. Detailed examination of the branched structure of konjac glucomannan. Agr. Biol. Chem. 1980, 44, 245-252.

4. Zhang, Y.Q.; Xie, B.J.; Gan, X. Advance in the applications of konjac glucomannan and its derivatives. Carbohydr. Polym. 2005, 60, 27-31.

5. Francis, S.; Kumar, M.; Varsheny, L. Radiation synthesis of superabsorbent poly(acrylic acid)carrageenan hydrogels. Radiat. Phys. Chem. 2004, 69, 481-486.

6. Wang, J.P.; Chen, Y.Z.; Ge, X.W.; Yu, H.Q. Gamma radiation-induced grafting of a cationic monomer onto chitosan as a flocculant. Chemosphere 2007, 66, 1752-1757.

7. Biswal, J.; Kumar, V.; Bhardwaj, Y.K.; Goel, N.K.; Dubey, K.A.; Chaudhari, C.V.; Sabharwal, S. Radiation-induced grafting of acrylamide onto guar gum in aqueous medium: Synthesis and characterization of grafted polymer guar-g-acrylamide. Radiat. Phys. Chem. 2007, 76, 1624-1630.

8. Pandey, P.K.; Srivastava, A.; Tripathy, J.; Behari, K. Graft copolymerization of acrylic acid onto guar gum initiated by vanadium (V)-mercaptosuccinic acid redox pair. Carbohydr. Polym. 2006, 65, 414-420.

9. Huacai, G.; Wang, P.; Dengke L. Graft copolymerization of chitosan with acrylic acid under microwave irradiation and its water absorbency. Carbohydr. Polym. 2006, 66, 372-378.

10. Vahdat, A.; Bahrami, H.; Ansari, N.; Ziaie, F. Radiation grafting of styrene onto polypropylene fibres by a $10 \mathrm{MeV}$ electron beam. Radiat. Phys. Chem. 2007, 76, 787-793.

11. Gupta, B; Scherer, G. Proton exchange membranes by radiation-induced graft copolymerization of monomers into Teflon-FEP films. Chimia 1994, 48, 127-37.

12. Abu, J.O.; Duodu, K.G.; Minnaar, A. Effect of $\gamma$-irradiation on some physicochemical and thermal properties of cowpea (Vigna unguiculata L. Walp) starch. Food. Chem. 2006, 95, 386-393.

13. Molins, R.A; Motarjemi, Y.; Kaferstein, F.K. Irradiation: a critical control point in ensuring the microbiological safety of raw foods. Food Control 2001, 12, 347-356.

14. Chen, L.G.; Liu, Z.L.; Zhuo, R.X. Synthesis and properties of degradable hydrogels of konjac glucomannan grafted acrylic acid for colon-specific drug delivery. Polymer 2005, 46, 6274-6281.

15. Wang, F.; Zou, H.N.; Zhou, D.H.; Wu, G.H. Preparation and characterization of high water absorption resin based KGM-graft-AA. Nat. Prod. Res. Dev. (Chin). 2006, 18, 282-285.

16. Xu, Z.; Sun, Y.; Yang, Y.; Ding, J.; Pang, J. Effect of $\gamma$-irradiation on some physiochemical properties of konjac glucomannan. Carbohydr. Polym. 2007, 70, 444-450.

17. Chapiro A. Preparation des copolymers greffes du polytetrafluoroethylene (Teflon) par viie radiochimique. J. Polym. Sci. 1959, 34, 481-501.

18. Flory, P.J. Principles of Polymer Chemistry. Cornell University Press: Ithaca, NY, 1953.

19. Luo, W.; Zhang, W.; Chen, P.; Fang, Y. Synthesis and properties of starch grafted poly[acrylamide-co-(acrylic acid)]/montmorillonite nanosuperabsorbent via $\gamma$-ray irradiation technique. J. Appl. Polym. Sci. 2005, 96, 1341-1346. 
20. Nishii, M.; Hayashi, K. Solid-state polymerization. Annu. Rev. Mater. Sci. 1975, 5, 135-149.

21. Nagasawa, N.; Mitomo, H.; Yoshii, F.; Kume, T. Radiation-induced degradation of sodium alginate. Polym. Degrad. Stab. 2000, 69, 279-285.

22. Relleve, L.; Nagasawa, N.; Luan, L.Q.; Yagi, T.; Aranilla, C.; Abad, L.; Kume, T.; Yoshii, F.; dela Rosa, A. Degradation of carrageenan by radiation. Polym. Degrad. Stab. 2005, 87, 403-410.

23. Prawitwong, P.; Takigami, S.; Phillips, G.O. Effect of $\gamma$-irradiation on molar mass and properties of konjac mannan. Food Hydrocolloid. 2007, 21, 1362-1367.

24. Molotkov, V.A.; Kurlyankina, V.I.; Klenin, S.I. Investigation of the kinetics of graft copolymerization of acrylamide with cellulose in the presence of trivalent cobalt salts. Polym. Sci. 1972, 14, 2890-2898.

25. Blundell, D.J.; Osborn, B.N. The morphology of poly(aryl-ether-ether-ketone). Polymer 1983, 24, 954-960.

26. Yu, H.Q.; Huang, Y.H.; Ying, H.; Xiao, C.B. Preparation and characterization of a quaternary ammonium derivative of konjac glucomannan. Carbohyd. Polym. 2007, 69, 29-40.

27. Vieria, M.C.; Gil, A.M. A solid state NMR study of locust bean gum glatactomannan and Konjac glucomannan gels. Carbohyd. Polym. 2005, 60, 439-448

28. Katsuraya, K.; Okuyama, K.; Hatanaka, K.; Oshima, R.; Satoc, T.; Matsuzaki, K. Constitution of konjac glucomannan: chemical analysis and ${ }^{13} \mathrm{C}$ NMR spectroscopy. Carbohydr. Polym. 2003, 53, 183-189.

29. Silva, D.A.; Paula, R.C.M.; Feitosa, J.P.A. Graft copolymerization of acrylamide onto cashew gum. Eur. Polym. J. 2007, 43, 2620-2629.

30. Ogawa, K.; Yui, T.; Mizuno, T. X-Ray-diffraction study of glucomannans and their acetates. Agr. Biol. Chem. 1991, 55, 2105-2111.

31. Tripathy, T.; Pandey, S.R.; Karmakar, N.C.; Bhagat, R.P.; Singh, R.P. Novel flocculating agent based on sodium alginate and acrylamide. Eur. Polym. J. 1999, 35, 2057-2072.

32. Xiao, C.B.; Lu, Y.S.; Zhang, L.N. Preparation and physical properties of konjac glucomannanpolyacrylamide blend films. J. Appl. Polym. Sci. 2001, 81, 882-888.

33. Garnett, J.L.; Jakiewicz, S.V.; Sangster, D.F. Mechanistic aspects of the acid and salt effect in radiation grafting. Radiat. Phys. Chem. 1990, 36, 571-579.

34. Ducouret, C.; Betz, N.; Le Moel, A. Study of the molecular weight distribution of polystyrene grafted by means of swift heavy ions. J. Chim. Phys. 1996, 93, 70-77.

Sample Availability: Available from the authors.

(C) 2008 by MDPI (http://www.mdpi.org). Reproduction is permitted for noncommercial purposes. 